\title{
ESTOU MAIS SEGURO NA MINHA CIDADE DO QUE OS OUTROS: IDENTIDADE COM O LUGAR E OPTIMISMO COMPARATIVO NA PERCEPÇÃO DE RISCOS URBANOS ${ }^{1}$
}

\author{
Maria Luísa Lima² \\ Sibila Marques ${ }^{3}$ \\ Susana Batel ${ }^{4}$ \\ Sandra Carvalho \\ Juana Haldon ${ }^{6}$ \\ Stephanie Pornin ${ }^{7}$
}

Resumo: Esta pesquisa pretendeu estudar o optimismo comparativo face aos riscos urbanos e explorar o papel que a identificação com a cidade de residência tem neste processo. $\mathrm{O}$ estudo foi realizado através de um inquérito a 156 estudantes universitários, residentes em Lisboa, Huelva e Lyon.

Os resultados revelaram a existência de optimismo comparativo face a todos os riscos urbanos considerados (carteirismo, assalto da casa, terrorismo e queda de avião): os riscos para o próprio foram sempre considerados inferiores aos riscos para os outros. No entanto, os residentes consideram-se menos vulneráveis do que os residentes de outras cidades (comparação exo-grupal) em particular no caso dos riscos graves e incontroláveis (terrorismo e queda de avião), sendo menos optimistas relativamente aos riscos menos graves e controláveis (assalto e carteirismo). Verificou-se que, no caso dos riscos urbanos incontroláveis, os inquiridos mais identificados com a sua cidade de residência revelam maior optimismo comparativo face aos residentes de outras cidades.

Palavras-chave: optimismo comparativo, riscos urbanos, identificação com o lugar.

\footnotetext{
${ }^{1}$ Esta pesquisa foi realizada com base nas ideias desenvolvidas no Workshop "Place identity and risk perception in the urban context" realizado na Escola de Verão em Psicologia Ambiental "Urban Spaces and Anomic Behaviours", uma inciativa conjunta do Departamento de Psicologia Social e das Organizações do ISCTE e do Laboratoire de Psychologie Environmentale de l'Université René Descartes (Paris V), que decorreu no ISCTE em Julho de 2005, com o apoio da FCT, do Centro de Investigação e de Intervenção Social do ISCTE e das Acções bilaterais Luso-Francesas. Este artigo não teria sido possível sem os contributos dos restantes elementos que participaram neste workshop: Elena Bilotta, Fabrizio Ventimiglia, Inês Alves, Rita do Carmo, Sandra Silva e Zulema Coronel.

2, 3, 4, 5 Instituto Superior de Ciências do Trabalho e da Empresa, Lisboa.

${ }^{6}$ Universidad de Huelva.

7 CitiZen, França.

PSICOLOGIA, Vol. XXI (2), 2007, Edições Colibri, Lisboa, pp. 45-58.
} 
I'm safer than the others in my town: place identity and comparative optimism in the perception of urban risks (Abstract): The goal of this study was to investigate comparative optimism regarding urban risks and to explore the role of identification with the city of residence in this process. Our sample included 156 university students from Lisbon, Huelva and Lyon.

Results revealed the occurrence of comparative optimism regarding all urban risks considered (pick pocketing, burglary, terrorism and airplane fall). As expected, risks for self were always considered inferior than risk for others. However, this effect was influenced by risk characteristics and degree of city identification. Participants considered themselves less vulnerable than residents in other cities (outgroup comparison) particularly in the case of severe and uncontrollable risks (terrorism and airplane fall) whereas they were less optimistic in the case of less severe and more controllable risks (burglary and pick pocketing). Regarding identification, we found that in the case of controllable urban risks, more identified individuals revealed more comparative optimism than those less identified.

Key-words: comparative optimism, urban risks and place identity.

\section{Introdução}

As questões da segurança são extremamente relevantes nas cidades, actuais e a investigação tem mostrado que o sentimento de vulnerabilidade é sensível a variáveis socio-cognitivas e ambientais. Este estudo testa algumas destas variáveis associadas à percepção dos riscos urbanos.

A Psicologia Social tem mostrado que as avaliações que fazemos sobre o mundo têm sempre implícitas comparações (Festinger, 1954; Moscovici, 1984), e essa perspectiva também se aplica à percepção de riscos (Joffe, 1999). Um dos fenómenos mais conhecido a este propósito designa-se por optimismo comparativo, isto é, a tendência a considerar o futuro do próprio como mais positivo do que o dos outros (Weinstein, 1980). Este efeito só recentemente foi aplicado aos riscos ambientais por Pahl e colaboradores (2005), mas não conhecemos estudos sobre este fenómeno relativamente aos riscos urbanos.

Neste estudo, procuramos explorar ainda algumas variáveis moderadoras deste fenómeno. Existem alguns estudos que demonstram uma relação entre a percepção de riscos ambientais e a identificação com o lugar. A este propósito, Bonaiuto e colaboradores (1996) concluíram que os indivíduos com uma forte identidade local minimizam os riscos ambientais na zona a que pertencem. Por sua vez, Lima (1997) verificou que os inquiridos mais identificados com a sua cidade percebiam menores riscos de sofrerem um terramoto do que os inquiridos menos identificados. No mesmo sentido, Lima e Marques (2005) verificaram que os inquiridos com maiores níveis 
de identificação com o lugar tinham níveis mais baixos de percepção de risco em relação à actividade de uma incineradora próxima.

No entanto, estes estudos sobre a identificação com o lugar e a percepção de riscos ambientais têm sido realizados em relação à percepção de risco para o próprio e não têm considerado o estudo do optimismo comparativo, ou seja, de que modo os riscos são percebidos por comparação a outros. O presente estudo pretende contribuir para o esclarecimento desta questão. Neste sentido, pretende estudar a relação entre o optimismo comparativo face a riscos urbanos e a influência da identificação com a cidade de residência. Especificamente, pretendemos avaliar de que modo a identificação com a cidade de residência afecta o optimismo comparativo relativamente a dois alvos: os residentes da mesma cidade (endogrupo) e os residentes de outra cidade semelhante (exogrupo).

Até ao momento, estudos anteriores revelaram que o optimismo comparativo é moderado pela pertença grupal do alvo (de ser do endogrupo ou do exogrupo do respondente). Neste sentido, espera-se que, em contextos em que a pertença ao endogrupo está saliente, o optimismo comparativo relativamente aos membros do endogrupo diminua e aumente o optimismo comparativo face aos membros do exogrupo (Harris, Middleton, \& Joiner, 2000). Uma vez que a identificação com uma determinada categoria social reflecte a prontidão do indivíduo para se auto-categorizar enquanto membro de um grupo (Spears, Doosje \& Ellemers, 1999; Turner, 1999), espera-se ainda que a identificação com a cidade de residência acentue este efeito, isto é, que diminua o optimismo comparativo em relação a residentes da mesma cidade e o aumente em relação a membros de "outras" cidades.

O presente estudo centra-se, então, nos riscos urbanos e pretende têstar as seguintes hipóteses:

Os residentes irão manifestar optimismo comparativo relativamente aos riscos urbanos $(\mathrm{H} 1)$;

O optimismo comparativo será mais elevado nas comparações com o exogrupo do que nas comparações com o endogrupo (H2);

Este último efeito será mais acentuado nos indivíduos mais identificados com a cidade de residência (H3).

\section{Método}

\section{Participantes}

Participaram neste estudo 156 indivíduos, divididos de forma equilibrada entre mulheres $(54 \%)$ e homens $(46 \%)$. Os dados foram recolhidos em 3 localidades diferentes: Lisboa (56 questionários), Huelva (50 questionários) e Lyon (50 questionários). 
Para minimizar as diferenças entre as amostras, controlaram-se diversas características dos participantes do estudo. Todos os respondentes considerados neste estudos eram estudantes universitários, com idades compreendidas entre os 18 e os 34 anos $(M=22,28 ; D P=3,53)$. De forma a certificarmo-nos da ligação dos respondentes à terra, controlámos ainda as seguintes variáveis: todos viviam actualmente na localidade estudada, e ou tinham lá nascido $(80 \%)$ ou viviam lá há bastante tempo. A média do tempo de residência dos 31 participantes que não nasceram no lugar estudado foi de 18,94 $(D P=4,02)$. Após termos eliminado os questionários que não cumpriam as condições de admissão no estudo, não se verificam diferenças estatisticamente significativas entre as 3 amostras ao nível do sexo $\left(\chi^{2}=1.83, g l=2, p=0,40\right)$, idade $(F(2,155)=0,24 ; p=0,79)$ ou tempo de residência na localidade $(F(2,155)=1,23 ; p=0,79)$.

Deste modo, não foi necessário controlar a origem dos questionários nas análises subsequentes.

\section{Instrumento}

No presente estudo, foi utilizado um questionário que, para além das questões demográficas iniciais, pretendeu medir as seguintes variáveis: identificação com a cidade de residência; distância social em relação aos residentes típicos da sua cidade de residência (endogrupo) e a residentes típicos de uma cidade semelhante à da sua residência (exogrupo); optimismo comparativo face a membros do endogrupo e do exogrupo relativamente a riscos urbanos; e experiência pessoal com esses riscos.

\section{Identificação com a cidade de residência}

Esta variável foi medida através de uma escala proposta por Lima (1994; 2005). Esta escala é composta por dois itens (e.g., "Sinto-me orgulhoso por viver em Lisboa" e "Sinto que pertenço a Lisboa") que são respondidos numa escala de 0 a $100 \%$. A escala foi adaptada a cada cidade em que o estudo foi realizado (i.e., Lisboa, Huelva ou Lyon).

Esta escala revelou boas qualidades psicométricas ( $\alpha$ de Chronbach $=0,80$ ) e permitiu o cálculo de um índice compósito referente à média das respostas aos dois itens.

Proximidade social dos membros do endogrupo e do exogrupo

Para medir a distância social relativamente aos membros típicos da cidade de residência (endogrupo) ou de outra cidade com características semelhantes (exogrupo) utilizámos uma tradução da escala IOSG (Inclusion of the Other in the Self scale for Group use) proposta por Harris et al (2000). Nesta escala, os participantes tiveram de escolher uma figura que 
representasse, de entre 9 possibilidades ("1", grande afastamento, até "9", sobreposição quase total), o grau em que consideravam que eram semelhantes aos residentes típicos da sua cidade do mesmo sexo e idade (Lisboa ou Huelva ou Lyon; endogrupo) e, posteriormente, a residentes típicos de uma cidade semelhante à sua, do mesmo sexo e idade (exogrupo).

Optimismo comparativo relativamente aos residentes típicos da sua cidade (endogrupo) e de cidades semelhantes (exogrupo)

O optimismo comparativo face a riscos urbanos foi medido através de uma medida indirecta, que consistiu na resposta a dois tipos de questões: a percepção de riscos urbanos para o próprio e a percepção de riscos urbanos para o "outro" (ambas respondido desde " 1 ", não existe risco, até "7", fortemente em risco). O "outro" foi, em primeiro lugar, considerado como o residente típico, da mesma idade e sexo, na cidade do participante (endogrupo) e, em segundo lugar, como o residente típico, da mesma idade e sexo, numa cidade semelhante à sua (exogrupo).

\section{Riscos urbanos considerados}

Uma vez que o optimismo comparativo varia em função da controlabilidade percebida do acontecimento em questão (Klein \& Helweg-Larsen, 2002; Bernardes, 2003), pré-testámos o grau de controlabilidade e de tipicidade de diversos riscos urbanos. Consideraram-se 2 riscos vistos como controláveis e típicos ${ }^{8}$ das localidades estudadas: roubo por carteiristas $\left(M_{\text {tipicidade }}=3,99 ; M_{\text {controlabilidade }}=4,7\right)$ e assalto à sua casa $\left(M_{\text {tipicidade }}=3,40\right.$; $\left.M_{\text {controlabilidade }}=4,8\right)$; dois outros riscos eram percebidos como incontroláveis e atípicos da cidade de residência: terrorismo $\left(M_{\text {tipicidade }}=1,75 ; M_{\text {controlabili- }}\right.$ dade $=2,2)$ e queda de um avião $\left(M_{\text {tipicidade }}=1,63 ; M_{\text {controlabilidade }}=2,1\right)$.

Para saber se os inquiridos já tinham experienciado pessoalmente algumas das quatro situações de riscos urbanos considerados, os participantes responderam numa escala dicotómica: "1", já viveu esta situação no passado, e "2", não viveu esta situação no passado. Como seria de esperar, os participantes têm maior experiência do primeiro grupo de riscos do que do segundo. No entanto, não há diferenças significativas de experiência, quando consideramos a cidade de origem dos participantes (ver Quadro 1).

\footnotetext{
${ }^{8}$ As avaliações foram feitas em escalas bipolares que variavam entre 1 (= nada) e 7 (= muitíssimo).
} 
Quadro 1 - Experiência pessoal com os riscos urbanos considerados

\begin{tabular}{lccccl}
\hline & $\begin{array}{c}\text { Lisboa } \\
\mathbf{\%}\end{array}$ & $\begin{array}{c}\text { Huelva } \\
\mathbf{\%}\end{array}$ & $\begin{array}{c}\text { Lyon } \\
\mathbf{\%}\end{array}$ & $\begin{array}{c}\text { Total } \\
\mathbf{\%}\end{array}$ & \\
\hline Carteirismo & 50 & 32 & 36 & 39 & $\chi^{2}=4,00 ;$ n.s. \\
Assalto & 16 & 12 & 28 & 18 & $\chi^{2}=4,59 ;$ n.s. \\
Terrorismo & 0 & 4 & 0 & 1 & n.s. \\
Queda de avião & 0 & 0 & 0 & 0 & n.s. \\
\hline
\end{tabular}

\section{Procedimento}

Todos os participantes responderam aos questionários através de autopreenchimento no início ou final de uma sala de aula de uma universidade em Lisboa, Huelva e Lyon. O questionário demorou cerca de 15 minutos a responder.

\section{Resultados}

Para testar a primeira hipótese, começámos por verificar a existência de optimismo comparativo nos nossos resultados (Quadro 2). Podemos constatar, em primeiro lugar, que a avaliação de riscos é muito diferente para os quatro tipos de acontecimentos, reflectindo a probabilidade de ocorrência destes fenómenos, tanto no caso da percepção de riscos para o próprio como para os outro9. Mas, como esperávamos, encontramos sistematicamente uma menor percepção de risco para o próprio do que para o outro. $\mathrm{O}$ teste $t$ para amostras emparelhadas mostra que, para os quatro riscos considerados, esta diferença de médias é significativa e correspondente às nossas hipóteses (Quadro 2). Realizou-se uma ANOVA 2 (alvo: próprio vs. outro) x 2 (controlabilidade: alta $v s$. baixa), com medidas repetidas em ambos os factores, que mostra o efeito principal do optimismo comparativo (efeito do alvo: $F(1,154)=22,49 ; p<0,001 ; \eta^{2}=0,127$ ) e o efeito principal do tipo de risco $\left(F(1,154)=298,026 ; p<0,001 ; \eta^{2}=0,659\right)$. O efeito de interacção não é significativo $(F(1,154)=0,161 ; p=0,689)$, o que quer di-

\footnotetext{
${ }^{9}$ Este indicador foi encontrado pela média das respostas dadas aos seguintes alvos "uma pessoa típica da sua cidade" e "uma pessoa típica de uma cidade de dimensão semelhante á sua”.
} 
zer que, para alvos não identificados intergrupalmente, o optimismo comparativo não é maior para eventos percebidos como típicos e controláveis.

Quadro 2 - Estatística descritiva da percepção de riscos urbanos para o próprio e para outro

\begin{tabular}{lcccccccc}
\hline & \multicolumn{9}{c}{ Próprio } & \multicolumn{2}{c}{ Outro } & & & \\
\cline { 2 - 6 } & Média & DP & Média & DP & $\boldsymbol{t}$ & $\boldsymbol{g} \boldsymbol{l}$ & $\boldsymbol{p}$ \\
\hline Carteirismo & 3,94 & 1,76 & 4,22 & 1,49 & $-3,357$ & 157 & 0,001 \\
Assalto & 3,44 & 1,40 & 3,69 & 1,17 & $-3,192$ & 156 & 0,002 \\
Terrorismo & 2,32 & 1,38 & 2,61 & 1,28 & $-3,844$ & 156 & 0,000 \\
Queda de avião & 1,85 & 1,18 & 2,14 & 1,12 & $-3,803$ & 157 & 0,000 \\
& & & & & & & \\
Riscos controláveis & 3,72 & 1,26 & 3,85 & 1,18 & & & \\
Riscos incontroláveis & 2,24 & 1,06 & 2,24 & 1,06 & & & \\
\hline
\end{tabular}

A análise do optimismo comparativo face a um membro típico do seu grupo ou de outra cidade não apresenta, porém, o mesmo perfil (Quadro 3).

No caso da comparação intragrupal, os participantes não se diferenciam do residente típico da sua cidade ao nível dos riscos de terrorismo $(t=-0,618, g l=157 ; p=0,54)$ ou queda de avião $(t=-1,480, g l=157$; $p=0,14)$, mas diferenciam-se no caso do carteirismo $(t=-4,273, g l=157$; $p=0,001)$ e do assalto $(t=-4,273, g l=156 ; p=0,001)$. No caso da diferenciação intergrupal o perfil das diferenças é praticamente simétrico: o optimismo comparativo observa-se de forma significativa apenas no caso do terrorismo $(t=-4,808, g l=157 ; p<0,001)$ e no da queda de avião $(t=-4,598, g l=157 ; p<0,001)$. A Figura 1 mostra o resultado da análise de variância 2 (tipo de risco: controlável vs. incontrolável) x 3 (alvo: próprio, endogrupo, exogrupo), com medidas repetidas em ambos os factores. 
Quadro 3 -Estatística descritiva da percepção de riscos urbanos para o próprio, para o residente típico da mesma cidade e de outra cidade

\begin{tabular}{lcccccc}
\hline & \multicolumn{2}{c}{ Eu } & \multicolumn{2}{c}{ Endogrupo } & \multicolumn{2}{c}{ Exogrupo } \\
\cline { 2 - 7 } & Média & DP & Média & DP & Média & DP \\
\hline Carteirismo & 3,94 & 1,76 & 4,24 & 1,69 & 4,19 & 1,54 \\
Assalto & 3,44 & 1,40 & 3,76 & 1,27 & 3,62 & 1,29 \\
Terrorismo & 2,32 & 1,38 & 2,36 & 1,31 & 2,85 & 1,54 \\
Queda de avião & 1,85 & 1,18 & 1,96 & 1,25 & 2,33 & 1,27 \\
& & & & & & \\
Riscos controláveis & 3,71 & 1,26 & 4,03 & 1,22 & 3,94 & 1,17 \\
Riscos incontroláveis & 2,09 & 1,06 & 2,17 & 1,10 & 2,61 & 1,19 \\
\hline
\end{tabular}

Figura 1 - Médias do optimismo comparativo intra e intergrupal: resultados em função do tipo de risco

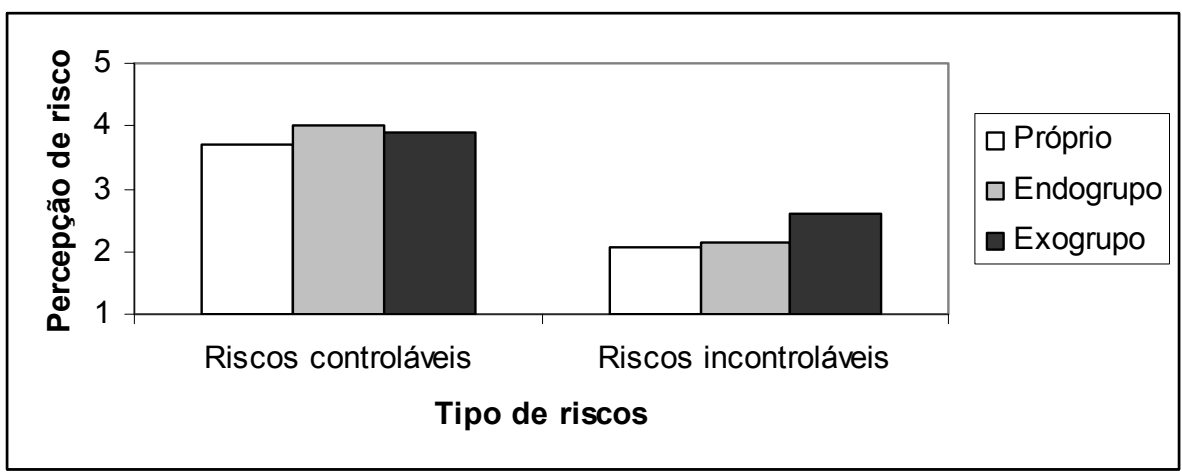

Os resultados mostram um efeito principal do tipo de risco $\left(F(1,306)=354,8 ; p<0,001 ; \eta^{2}=0,699\right)$, correspondendo a uma maior percepção de risco, no caso dos riscos controláveis $(M=3,89 ; E P=0,09)$ do que dos incontroláveis $(M=2,29 ; E P=0,08)$, e outro do tipo de alvo $\left(F(2,306)=17,5 ; p<0,001 ; \eta^{2}=0,175\right)$, que, como esperávamos na Hipótese 2, corresponde a níveis mais baixos de risco percebido para o próprio $(M=2,90 ; E P=0,08)$, seguidos do endogrupo $(M=3,09 ; E P=0,08)$ e com 
valores máximos para o exogrupo $(M=3,28 ; E P=0,08)$. No entanto, estes efeitos são qualificados com um efeito de interacção $(F(2,306)=17,7$; $p<0,001 ; \eta^{2}=0,210$ ), que mostra que o optimismo comparativo endogrupal é mais elevado para os riscos controláveis e típicos da localidade e o optimismo comparativo exogrupal é mais elevado para os riscos incontroláveis e atípicos. Este resultado inesperado mostra que distintividade positiva intergrupal é saliente para os riscos vistos como mais graves, enquanto que, para os riscos mais banais, se mantém a distintividade intragrupal.

Para verificar a H3, procurámos esclarecer o papel que a identificação com a cidade de residência tem com as estimativas de riscos urbanos para o próprio, para o membro do endogrupo e do exogrupo.

Antes de mais, analisámos a relação entre as duas medidas de ligação ao lugar, a identificação com o lugar e a distância social percebida com os membros do endogrupo e do exogrupo. Como se esperava, verificou-se uma correlação negativa e significativa entre a identificação com a cidade de residência e a distância social com os membros do endogrupo: quanto mais os inquiridos se identificam com a sua cidade de residência, mais se consideram próximos dos residentes típicos da sua cidade $(r=-0,29$; $p=0,00)$. Não se verificou uma relação significativa entre a identificação com a cidade de residência e a semelhança com os membros típicos do exogrupo $(r=0,08 ; p=0,27)$.

Realizámos uma análise de variância aos indicadores de optimismo comparativo, usando um design 2 (risco: controlável vs. incontrolável) x 3 (alvo: próprio, endogrupo, exogrupo) x 2 (identificação: baixa e alta), com medidas repetidas nos dois primeiros factores. Os resultados mostram, para além dos efeitos principais e da interacção alvo x risco que já tínhamos obtido anteriormente, um efeito de interacção triplo tangencialmente significativo $\left(F(4,302)=2,19 ; p<0,07 ; \eta^{2}=0,03\right)$. Estes resultados (Figura 2) indicam que o optimismo comparativo face ao exogrupo para os riscos graves e incontroláveis só se aplica no caso dos indivíduos identificados com a localidade: os riscos percebidos para o próprio $(M=2,19 ; E P=0,14)$ são semelhantes aos do endogrupo $(M=2,11 ; E P=0,15)$, mas significativamente inferiores aos do exogrupo $(M=2,59 ; E P=0,16)$; os mesmos indicadores para os indivíduos menos identificados mostram valores mais próximos para os 3 alvos (próprio: $M=1,98 ; E P=0,15$; endogrupo: $M=2,20 ; E P=0,15$; exogrupo $M=2,34 ; E P=0,16$ ). No caso dos riscos menos grãves e mais controlaveis, não há efeitos da identidade dos residentes na avaliação dos alvos. 
Figura 2 -Médias do optimismo comparativo intra e intergrupal em função do tipo de risco. Resultados por nível de identidade com a localidade onde residem
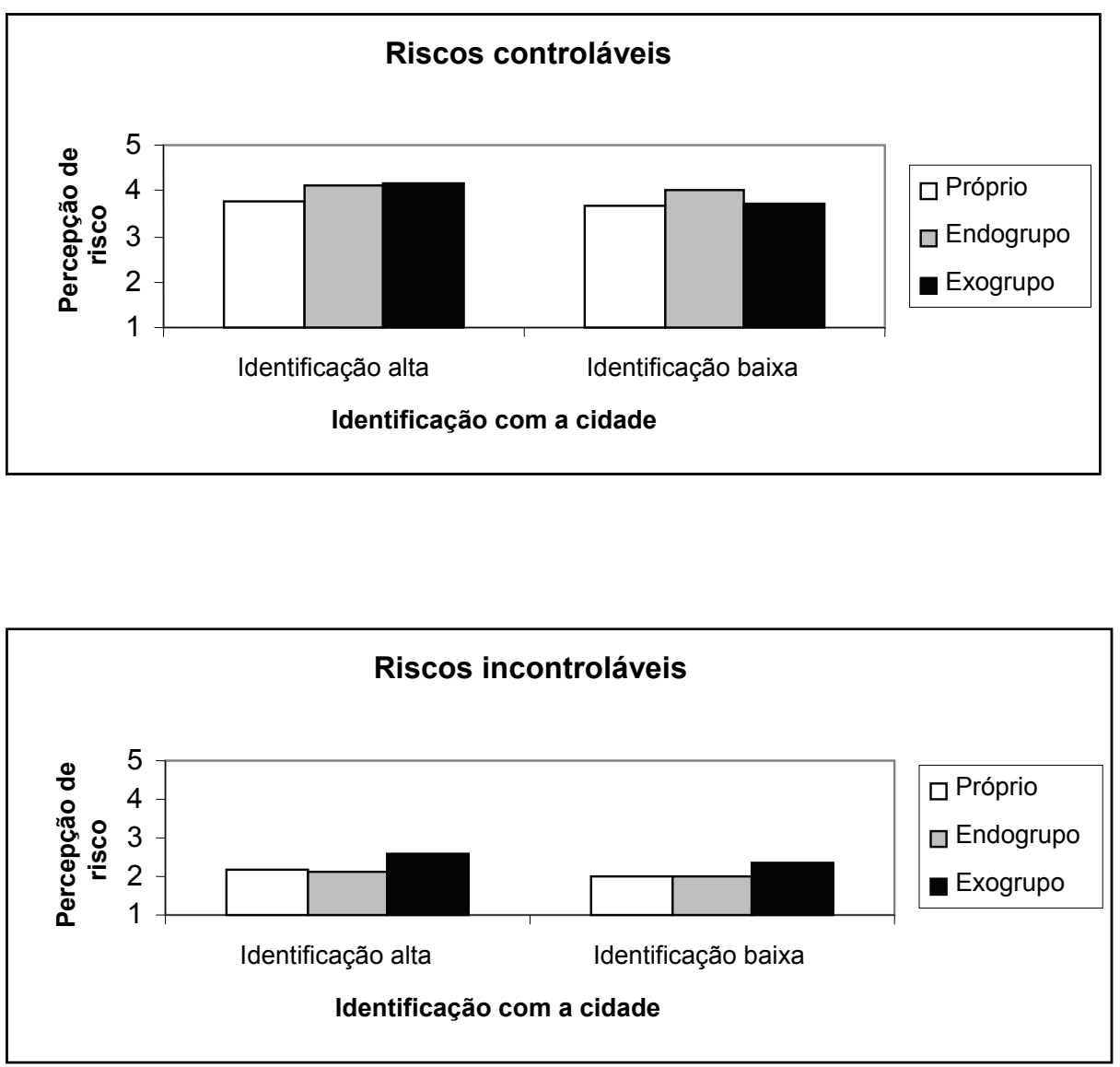

\section{Discussão}

De acordo com as hipóteses de estudo, esperávamos encontrar optimismo comparativo relativamente aos riscos urbanos (H1) e que ele fosse mais elevado nas comparações exogrupais do que nas comparações endogrupais (H2). Para além disso, e porque queríamos explorar o papel da identidade local no optimismo comparativo, esperávamos ainda que a maior identificação com a cidade de residência estivesse relacionada com a diminuição do optimismo comparativo face ao membro do endogrupo e aumento em relação ao membro do exogrupo (H3) 
A partir da análise dos resultados, verificou-se apoio empírico para a primeira hipótese: para qualquer dos riscos considerados, os indivíduos percebiam-se como menos vulneráveis do que os outros. Este resultado situa-se na linha de muitos outros trabalhos sobre o optimismo comparativo, face a riscos de saúde, e continua, para os riscos urbanos, os resultados obtidos por Pahl e colaboradores (2005) para os riscos ambientais. Isto é, também para o caso dos riscos urbanos podemos afirmar que os outros estão mais em risco do que nós, o que se pode resumir na frase: "estou mais seguro do que os outros na cidade".

Esperávamos ainda que o nível de optimismo comparativo fosse maior face ao exogrupo do que face ao endogrupo, mas esta hipótese foi apenas parcialmente confirmada. De facto, este padrão verificou-se para os riscos atípicos (raros, graves e incontroláveis), mas não para os riscos típicos (mais comuns, menos graves e percebidos como mais controláveis).

A este respeito, importa salientar que o padrão de resultados verificado salienta dois aspectos fundamentais que interagem na forma como os riscos urbanos são percebidos: a natureza dos riscos avaliados e o alvo de comparação.

O optimismo comparativo é um fenómeno multideterminado que funciona como um modo de regular a ansiedade e afectos negativos através da ideia de que os próprios estão em melhor situação do que os outros. No entanto, a expressão deste fenómeno depende de algumas variáveis moderadoras (Helweg-Larsen \& Shepperd, 2001; Klein \& Helweg-Larsen, 2002). Relativamente às características dos riscos, salientamos dois aspectos: a gravidade e a controlabilidade dos eventos. Por um lado, os estudos nesta linha de pesquisa têm demonstrado que a existência de optimismo comparativo é superior, no caso de riscos mais graves e indesejados (Kirsht, Haefner, Kegeles \& Rosenstock, 1966). Por outro lado, alguns estudos demonstram que o optimismo comparativo é superior, no caso dos eventos percebidos como controláveis pelos indivíduos (Bernardes, Lima \& Paulino, 2005; Klein \& Helweg-Larsen, 2002).

No presente estudo, o optimismo comparativo face a um "outro" indeterminado não varia em função das características dos riscos: riscos controláveis e típicos apresentam o mesmo nível de optimismo comparativo que os incontroláveis e atípicos. No entanto, o optimismo comparativo diferencia-se quando se considera a interacção das características do risco com a natureza do alvo que serve de comparação.

Relativamente a este resultado, importa considerar que a escolha de alvos de comparação é flexível e é realizada em função dos alvos que permitem obter uma maior positividade relativa (Helweg-Larsen \& Shepperd, 2001; Perloff \& Fetzer, 1986). Neste sentido, os nossos resultados indicam, pela primeira vez, que a natureza dos riscos poderá determinar o alvo de 
comparação intergrupal que é escolhido. Para riscos comuns, controláveis e pouco graves, a distintividade intragrupal e intergrupal é semelhante. Neste caso, o facto de o alvo de comparação pertencer ao exogrupo ou ao endogrupo parece não ser relevante. Estes são riscos que dependem de um controlo individual e, por essa razão, os indivíduos julgam que poderão estar melhor do que os restantes indivíduos independentemente de pertencerem à sua cidade ou a outras semelhantes.

No entanto, no caso de riscos mais graves e incontroláveis, a dimensão intergrupal assume um carácter central. De certo modo, este resultado poderá estar relacionado com o facto de os riscos mais graves (i.e., terrorismo e queda de avião) dependerem menos do controlo pessoal do que de um controlo social, ao nível macro de organização da cidade (neste sentido, os inquiridos poderão pensar "embora eu e os outros habitantes da minha cidade não possamos controlar o risco de terrorismo e queda de um avião, a minha cidade controla melhor esse risco do que outras cidades semelhantes"). Esta interpretação dos resultados é ainda congruente com o efeito encontrado de moderação da identidade (H3): o optimismo comparativo face aos membros do exogrupo acentua-se nos residentes mais identificados, quando avaliam riscos considerados incontroláveis e atípicos. Esta interpretação vai na direcção da proposta de Klein e Helweg-Larsen (2002), que salientam a importância de distinguir formas de controlo primário e secundário na compreensão do optimismo comparativo.

Tal como referido anteriormente, estes resultados enfatizam a relevância de se considerar a importância da dimensão intergrupal como determinante, sobretudo no caso de riscos graves e percebidos como incontroláveis individualmente. No caso dos riscos comuns, o grau de ligação à cidade não influencia a percepção desses riscos controláveis individualmente. No entanto, no caso de riscos graves e que não são controlados individualmente, a dimensão intergrupal assume uma relevância central. Neste sentido, são sobretudo os inquiridos mais identificados com a sua cidade que utilizam mais esta estratégia de diferenciação intergrupal para minimizarem os riscos relativamente a outras cidades.

Em termos gerais, os resultados obtidos salientam a importância de se considerarem variáveis psicossociais, na forma como os riscos urbanos são percebidos. Nomeadamente, salienta-se, neste estudo, a importância de considerar a influência do tipo de riscos que são avaliados, dos grupos a que pertencemos (a "nossa" cidade ou a cidade dos "outros") e o grau em que esses grupos são relevantes para a nossa identidade. Só deste modo podemos dar sentido às complexas estratégias identitárias na construção do sentimento de segurança: na "nossa" cidade estamos mais seguros do que os outros, face a riscos insignificantes; mas a "nossa" cidade é mais segura do que as outras quando os riscos são mesmo assustadores. 


\section{Referências}

Bernardes, S. (2003). O optimismo comparativo face à Saúde em Crianças e Adolescentes: diferenças etárias na sua expressão e relação com as crenças associadas ao controlo. Tese de Mestrado em Psicologia Social e Organizacional. Lisboa: Instituto Superior de Ciências do Trabalho e da Empresa.

Bernardes, S., Lima, M. L., \& Paulino, P. (2005). O optimismo comparativo face à saúde: da infância à adolescência. Psychologica, 39, 207-225.

Bonaiuto, M., Breakwell, G. M., \& Cano, I. (1996). Identity processes and environmental threat: The effects of nationalism and local identity upon perception of beach pollution. Journal of Community and Applied Social Psychology, 6, 157-175 .

Festinger, L. A. (1954). A theory of social comparison processes. Human Relations, 7, 117-140.

Harris, P. R., Middleton, W., \& Joiner, R. (2000). The typical student as an in-group member: Eliminating optimistic bias by reducing social distance. European Journal of Social Psychology, 30, 235-253.

Helweg-Larsen, M., \& Shepperd, J. (2001). Do moderators of the optimistic bias affect personal or target risk estimates? A review of literature. Personality and Social Psychology Review, 5, 74-95.

Joffe, H. (1999). Risk and "the other". Cambridge: Cambridge University Press.

Klein, C. T. F., \& Helweg-Larsen, M. (2002). Perceived Control and the Optimistic Bias: a Meta-Analytic Review. Psychology and Health, 17, 437-446.

Kirscht, J. F., Haefner, D. P., Kegeles, S. S., \& Rosenstock, I. M. (1966). A national study of health beliefs. Journal of Health and Human Behavior, 7, 248-254.

Lima, M. L. (1994). A Percepção do Risco Sísmico: Medo e Ilusões de Controlo. Tese de doutoramento apresentada no Instituto Superior de Ciências do Trabalho e da Empresa, Lisboa.

Lima, M. L. (1997). Earthquakes are not seen in the same way by everyone. Cognitive adaptation and social identities in seismic risk perception. In O. Renn (Ed.), Risk analysis and management in a global economy (vol. 2, pp. 181-201). Baden-Wurttemburg: Center of Technology Assessment.

Lima, M. L., \& Marques, S. (2005). Towards successful social impact assessment follow-up: A case study of psychosocial monitoring of a solid waste incinerator in the north of Portugal. Impact Assessment and Project Appraisal, 23, $227-$ -233 .

Moscovici, S. (Ed.). (1984). Psychologie sociale. Paris: Presses Universitaires de France.

Pahl, S., Harris, P. R., Todd, H. A., \& Rutter, D. R. (2005). Comparative optimism for environmental risks. Journal of Environmental Psychology, 25, 1-11.

Perloff, L., \& Fetzer, B. (1986). Self-other judgements and perceived vulnerability to. victimization. Journal of Personality and Social Psychology, 50 (3), 502-510 .

Shepperd, J. A., Carrol, P., Grace, J. \& Terry, M. (2002). Exploring the causes of comparative optimism. Psychologica Belgica, 42, 65-98. 
Spears, R., Doosje, B., \& Ellemers, N. (1999). Commitment and content of special perception. In N. Ellewers, R. Spears \& Doosje (Eds.), Social Identity: context commitment, content (pp. 59-83). Oxford: Blackwell Publishers.

Turner, J. C. (1999). Some current issues in research in social identiy and self-categorization theories. In N. Ellemers, R. Spears \& B. Doosje (Eds.), Social identity: Context, commitment, content (pp. 6-34). Oxford: Blackwell Publishers.

Weinstein, N. D. (1980). Unrealistic optimism about future life events. Journal of Personality and Social Psychology, 39, 806-820. 